\title{
Research on seeding agents to effect the sugar crystallization
}

\author{
Dorin Popa and Gheorghe Sarca \\ Oradea University, Faculty of Environmental Protection
}

\begin{abstract}
The quality of sugar is the decisive factor in ensuring the sugar commercializing through the conservation of old markets and expansion towards new markets. The sugar quality is also the key element which determines the price maintenance in according with the expenditures performed or even the diminution of the price as a consequence of the quality lessening.
\end{abstract}

Key words: sugarbeet,technological process,cristalisation ,isopropyl alcool

\section{INTRODUCTION}

Securing the technological process efficiency is mainly done through the following:

- Decreasing to the minimum level the sugar amount re-circulated from the $1^{\text {st }}$ step of crystallization to the $2^{\text {nd }}$ one;

- Decreasing to the minimum level the sugar amount re-circulated from the $2^{\text {nd }}$ step of crystallization to the $3^{\text {rd }}$ one;

- Permanent preoccupation to avoid the drawing in the molasses of those small crystals that can pas through the loops of the centrifugal sieves;

- Obtaining the top quality sugar mainly defined by:

- $\quad$ solution colouring, sugar-in-water solutions transparency

- polarisation, which shows the amount of saccharose content out of the crystal sugar;

- humidity and the content of conductor-metrical ashes;

- the size the shape and the crystal homogeneity;

all these are being worldwide achieved by the way in which the crystallizing and shaping technology regarding the emerged crystals is conducted.

\section{MATERIALS AND METHODS}

\section{Methods for starting the saccharose crystallization}

There are well-know 3 methods for saccharose crystallization starting:

- $\quad$ seeding in the field of supersaturating metastable by inserting desired quantity of crystallizing germs in the concentrated syrup;

- crystallization centres formation by a thermal shock effect in the super-saturation intermediate level;

- spontaneous or natural crystallization in the unstable field.

The main method for starting the saccharose crystallization out of its super-saturated solutions takes place through the seeding method. The seeding is the preliminary condition for the transition to the boiling and to the controlled and automatic crystallization of the sugar as only in this way one can reach to the process reproducibility to the

\section{Seeding with powder sugar}

The quality of the obtain sugar relies, and especially the homogeneity of the crystals obtained, the shape of the crystals, the occurrence and the conglomerates content, all depend on the way the seeding is done. In Romania, powder sugar has been used for a very long time and it is still being used as seeding agent. The use of powder sugar in alcoholic suspension presents certain advantages as against the use of only powder sugar as such. The advantages of the seeding with powder sugar are the following:

- one can accurately establish the number of germs contained in the seeding solution which leads us to the awareness of the total number of germs introduced in the vacuum apparatus;

- one can ensure the uniformity of the germs from their size point of view;

- one can achieve the advanced dispersal of the sugar particles out of the alcoholic suspension. This dispersal attracts itself an improvement of the sugar' quality both from its crystal aspect point of view and from chemical point of view. Due to the advanced disposal degree, the conglomerate formation is avoided. The crystal sugar conglomerates are the formations resulted from the sugar crystals bonding. During their shaping these conglomerates include "mother" syrup. This fact leads to the augmentation of the conductor-metrical and carbonic ashes in the crystal sugar; 
- $\quad$ achievement of a better homogenising of the crystallization germs within the supersaturated syrup mass;

- the opportunity for preparing a higher amount of powder sugar alcoholic suspension due to its long-lasting stability;

replacing the sugar powder, consider "classical agent for seeding" which is due also to the humidity contained in the sugar crystals which is freed through the crystals' milling which enhance the crystal sugar tendency to form in the lob run of crystal lumps.

\section{A method for sugar powder suspension preparation in isopropyl alcohol}

In the specialised literature several method for the preparing the alcoholic suspension for sugar crystallisation seeding are mentioned. These methods have been studied within the Sugar Research and Engineering Laboratory from the Food Chemistry Institute Bucharest, but one can adapt them to our working methods. The method adopted consists in sugar milling directly in isopropyl alcohol.

The method is two-phased one, namely:

- $\quad$ obtaining the base suspension;

- $\quad$ the base suspension dilution and obtaining the seeding suspension.

On the basis of the experimental results obtained in the laboratory, which consist in sugar milling in isopropyl alcohol in a 5.01 milling machine on balls working on rolling and having the a 46 rotations/min spin several working conditions have been established which are presented bellow.

Obtaining the base suspension

- $\quad$ for a charge on employ:

- 100 grams of crystal sugar having a granulation of $0.2-0.8 \mathrm{~mm}$;

- $200 \mathrm{ml}$. of isopropyl alcohol;

- $\quad$ about 160 porcelain balls having a diameter of $30 \mathrm{~mm}$ with a total mass of $1.0 \mathrm{~kg}$;

- $\quad$ about 600 porcelain balls having the diameter of $12 \mathrm{~mm}$ with a total mass of $1.0 \mathrm{~kg}$;

- $\quad$ the milling period is 1 hour.

Obtaining the seeding suspension Out of the base suspension thus obtained, the seeding suspension is prepared through its dilution in isopropyl alcohol in a 1:200 ratio.

Maturing the seeding suspension The thus prepared powder sugar suspension in isopropyl alcohol, called seeding suspension, is let to repose for a maturing period of 4 weeks. In the meanwhile, the germs smaller than $1 \mathrm{~mm}$ are being dissolved while the sugar dissolved crystallizes on larger particles. Subsequent to this maturing period equilibrium is established while the number of sugar particles from the seeding suspension actually remains constant.

Criteria for ensuring the reproducibility of the seeding suspension quality In order to always obtain a suspension with appreciatively the same number of particles on the mass unit, one recommends the observance of the following measures:

- using the sugar with the same granulation for milling namely the sugar of $0.2-0.8 \mathrm{~mm}$, deprived of powder or conglomerates;

- maintaining the ratio "sugar parts" against the "isopropanol parts". As smaller quantity of sugar is used on a isopropanol unit as brutish the sugar from the obtained suspension will be. The best results are obtained using for a part of granulation sugar of $0.2 \mathrm{~mm}-0.8 \mathrm{~mm}$ or a ratio of 2.0-2.2 parts of isopanorol;

- $\quad$ observing the milling period for the same mill machine functioning conditions (criteria). The milling period prolonging does not imply a better milling.

\section{A method for powder sugar preparation in ethyl alcohol}

The permanent tendency to improve the preparation process of powder sugar suspension preparation during the saccharose crystallization striking, in the sense to improve the dispersal technique in solvent, led to a new preparation method of the seeding suspension which is based on saccharose reduced solubility in ethyl alcohol. This method consists in sugar precipitation from a saturated solution in ethyl alcohol. The specialised literature recommends a similar method which consists in precipitating of a 21 grams of sugar which is found in a saturated solution at $55^{\circ} \mathrm{C}$, a solution of $28^{\circ}$ Brix concentration or $28 \%$ in ethyl alcohol.

Preparing the base suspension The laboratory experiments accomplished on the milling machine based on balls allowed the elaborating of the following working method:

- $\quad$ one introduce 21 grams ethyl alcohol in the milling machine on which one pour, in a thin stripe, a $28 \%$ sugar solution which contains 420 grams of powder sugar;

- one activate the milling machine in rotating (spin) motion. The milling functioning period is of 30 minutes during which the sugar crystallizing under the suspension form;

- after the sugar crystallized under suspension, the suspension is pulled out of the milling machines on balls and it is let for reposing for 10 up to 15 minutes; 
- $\quad$ one replace the supernatant liquid with a new fresh film (layer) of ethyl alcohol;

- the mixture of ethyl alcohol and sugar is again introduced in the milling machine on balls which is activated for another 10 minutes.

The sugar suspension thus prepared can be used after a minimum period of two days, as $\mathrm{n}$ the meanwhile it becomes stable. In order to be preserved for a long period of time, the film of supernatant alcohol is replaced one more time with fresh ethylic alcohol.

Form the point of view of shape and size, the sugar crystals of the suspension thus prepared are very homogeneous.

Preparing the seeding substance Out of the base suspension one prepare the seeding suspension through the dilution of 1,200 in isopropyl alcohol. In comparison with the method described above (at point 15.4), the current method which employs ethyl alcohol for base suspension preparation is easier to be executed.

Establishing the number of germs introduced within a "decoction" The crystal sugar obtaining of a certain granulation depends on the number of germs introduced at a single seeding in the vacuum apparatus. Before reaching to this, one establish the number of germs from the seeding suspension and then one calculate the required quantity (amount) function of the size of the sugar crystals which is sought to be obtained. For establishing the number of germs in the sugar suspension there are used the following methods:

- the microscopically method;

- $\quad$ the sedimentation method;

- $\quad$ the sieving (sifting) method

In this purpose one can employ; the sedimentation balance, the Andreasen dropper or the Coulter counter. Bearing in mind the number of germs which are contained in the seeding suspension, we calculate the necessary amount to effect crystallization. In practice, a surplus of germens is employed in experiment due to the different super-saturation in the component parts of the vacuum apparatus, especially in the case in which this is not endowed with a mechanical system for stirring up. The exceeding quantity of crystallizing germs depends on other factors such as, foe example:

- $\quad$ the crystallizing step at which the striking (effecting) is been done;

- $\quad$ the constructive type (model) and the capacity of the vacuum apparatus;

- the pressure of the heating steam.

In the case of the A product the specialised literature recommends the use of a crystallization germs surplus of $30-50 \%$ in comparison with the theoretical necessary. The crystallizing efficaciousness (rated capacity) from a gross mass is theoretically know as it can be calculated using the calculation formula based on gross mass purity and of the inter-crystalline syrup and on the dry-mass content of the gross mass. The calculation formula for the crystals extracted of gross mass' efficaciousness (rated capacity) is the following:

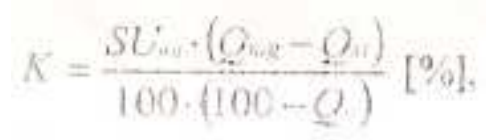

where:

$\mathrm{SU}_{\mathrm{mg}}$ is the content of dry-mass of the gross-mass în ${ }^{\circ}$ Brix:

$\mathrm{Q}_{\mathrm{mg}} \mathrm{g}$ - purity of the gross mass in \%

$\mathrm{Q}_{\mathrm{si}}$ - purity of the inter-crystalline syrup, meaning the purity of the green syrup 1 in $\%$

For the crystal mass the following formula is used:

$\mathrm{p}=\mathrm{d}^{3} \mathrm{X} 1.1[\mathrm{mg}]$,

where:

$\mathrm{p}=\mathrm{is}$ the desired individual mass of a single sugar crystal, in mg:

$\mathrm{d}$ - is the web dimension of the whole corresponding to the desired size of the sugar crystal, in mm;

The specific mass of the crystal sugar in non-settled way

For example if we want to obtain sugar crystals with the web dimension of the whole sized of $0,7 \mathrm{~mm}$, the calculation is done as following:

- the desired individual mass of a single sugar crystal which will be obtained after crystallization is calculated using the following formula:

$0.7^{3}$ X $1.1=0.3773 \mathrm{mg} ;$

a single tone of crystal sugar obtained through crystallization, formed through crystallization and constituted from crystals with an individual mass of $0.3773 \mathrm{mg}$, will contain:

$$
1000000000: 0.3773=28 \times 10^{8} \text { crystals }
$$

This number represents also the number of crystallization germs of which must be, theoretically, introduced in the vacuum apparatus for crystallization striking (initiating) and to obtaining of the tone of crystal sugar. 
If we take into consideration:

- $\quad$ the vacuum apparatus capacity 501;

- the crystal efficaciousness (output rate) $45 \%$ we can calculate:

- the amount of crystal sugar which must be obtained at a single decoction namely:

$50 \times 0.45=22.5$ tones

- the number of crystals contained by the decoction thus obtained is:

$$
22.5 \times\left(28-10^{8}\right)=630 \times 10^{8} \text { crystals }
$$

\section{RESULTS AND DISCUSSIONS}

This number, namely $630 \times 10^{8}$ represents also the number of germs which must be introduced in the vacuum apparatus at crystal initiation (striking).

If we consider the fact that through the previous measurements is has been established that in $100 \mathrm{ml}$ of seeding suspension there are $325 \times 10^{9}$ crystallization germs, an amount of $20 \mathrm{ml}$ seeding suspension calculated according to the following formula $\left(630-10^{8}\right):\left(325-10^{9}\right)=20 \mathrm{ml}$ will be required.

Taking into account the exceeding amount of crystallizing germs, $30-50 \%$ sugar the real amount of seeding suspension, introduced in the vacuum apparatus at a single decoction with $0.7 \mathrm{~mm}$ crystals, is calculated using the following formula:

$$
20 \mathrm{X}(1.30-1.50)-20 \mathrm{ml}
$$

This is the way of seeding suspension preparation and also the way to calculate the seeding suspension volume required to initiate (strike) the crystal sugar in the above mentioned conditions and data.

\section{REFERENCES}

1. Banu C., 1992 - "Progrese tehnice, tehnologice ă i ă tiină ifice în industria alimentară”, Ed. Tehnică, Bucuresti.

2. Beceanu Dumitru, Chira Adrian, 2003 - "Tehnologia produselor agricole”, Ed. Economică, Bucuresti.

3. Sarca Gheorghe, 2004 - "Materii prime vegetale”, Ed. Univ. din Oradea.

4. Sarca Gheorghe, 2009 - "Tehnologia zaharului", Ed. Univ. din Oradea

5. International Sugar Journal, 2010 May 